\title{
Identification of textile fibers by terahertz time domain spectroscopy
}

\author{
TONG Minming, CAO BingHua, CAI Li, TONG Ziyuan \\ School of Information and Electrical Engineering, China University of Mining and Technology \\ Xuzhou,China,221116 \\ e-mail: caobinghua2004@163.com
}

\begin{abstract}
A method to discriminate textiles based on terahertz time-domain spectroscopy (THz-TDS) is proposed, and some commonly used synthetic fibers are investigated to study its feasibility. Their absorption spectra are measured using the THzTDS system. The experimental results show that terylene, polyamide and vinylon each have a characteristic absorption peak at $0.98 \mathrm{THz}, 1.51 \mathrm{THz}$ and $1.16 \mathrm{THz}$ in their absorption spectra respectively. Simultaneously, aromatic polyamide fiber, acrylic fiber and Tencel fiber have no characteristic peaks and still differ from each other very much. The research results show that it is feasible to apply terahertz time domain spectroscopy into the identification of synthetic fibers. Still it indicates that terahertz technique is a promising one for the detection of textiles.
\end{abstract}

Keywords- terahertz, textile, identification

\section{INTRODUCTION}

In textile manufacturing management and product analysis of textile, it is essential to identify fiber in different state. The materials of textile products are identified by the fiber's figure, chemical composition or the scent of gas when it is burning. However, most of these traditional methods are complex ${ }^{[1-3]}$. Moreover some of them will produce lots of harmful gases and pollute the working environment. Therefore, the textile industry requires a simple, rapid and reliable method for textile identification.

Terahertz $(\mathrm{THz})$ wave is the electromagnetic spectrum situated between microwave and infrared wave. Over the last decade, advance in ultrafast pulse laser has led to coherent generation and detection of sub-picoseconds, broasband $(0.1 \mathrm{THz}-10 \mathrm{THz})$ pulses ${ }^{[4]}$. Terahertz wave has some unique properties. First, terahertz wave has a long wavelength so it can be less affected by scattering. Second, terahertz wave has an interesting property that it can easily transmit through many nonmetallic materials, such as plastic, cardboard, wood and glass which makes it an excellent alternative for nondestructive detection of these materials. The third important property for terahertz wave is its low photon energy, which makes it a safe ray for human beings ${ }^{[5]}$. Terahertz time domain spectroscopy (THz-TDS) is one of the most popular terahertz techniques. In THz-TDS system, terahertz wave is detected by optical sampling, and it allows direct measurement of the terahertz transient electric field, i.e., both the amplitude and phase are obtained simultaneously ${ }^{[6]}$. Moreover, THz-TDS can obtain a SNR as high as 1010 because optical sampling detection makes the detected signal insensitive to the background noise. Up to now, THz-TDS technique has been studied and applied in many researches, such as gas spectroscopy ${ }^{[7-8]}$, explosive identification $^{[9-10]}$, pharmaceutics ${ }^{[1-12]}$, T-ray imaging ${ }^{13-14]}$, and so on. However, in the process of textile identification, few researches about the application of terahertz technique are published.

In this work, terahertz time domain spectroscopy is applied to measure the $\mathrm{THz}$ response of textile fibers. Six kinds of textile fiber, terylene, polyurethane fiber, polyamide fiber, acrylic fibers, tencel and vinylon are selected to verify the feasibility of applying THz-TDS into textile identification.

\section{EXPERIMENT}

\section{A. Setup}

Figure 1 shows a typical terahertz time domain spectroscopy apparatus, a Ti: sapphire laser with $68 \mathrm{fs}$ pulse duration, $800 \mathrm{~nm}$ in central wavelength, $82 \mathrm{MHz}$ in repetition rate, and an output power of $980 \mathrm{mw}$ is used. The laser beam is divided into two parts: a pump and a probe. The pump laser beam is illuminated onto a large-aperture GaAs photoconductive antenna to generate terahertz wave. The generated terahertz wave is collimated and focused on the sample by a pair of parabolic mirrors (PM). After being transmitted through the sample, the terahertz wave is collimated and focused again onto a ZnTe electro-optic (EO) crystal by the second pair of parabolic mirrors. The probe beam is coincident with the terahertz wave to detect polarization changes due to Pockel's effect of terahertz wave. The linearly polarized probe beam experiences the polarization change due to a birefringence by the terahertz wave in $\mathrm{ZnTe} \mathrm{EO}$ crystal. With varying time delay between the terahertz wave and probe beam, the entire terahertz wave is traced and its waveform decoded. After amplified by a lock-in amplifier, the measured signal is put into the computer to be further processed. In order to minimize absorption of water vapor and increase the signalto-noise ratio (SNR), $\mathrm{THz}$ beam path is masked by a box purged with nitrogen (dashed line frame in Fig. 1).

This work is supported by China Postdoctoral Science Foundation (No. 20100481177), Jiangsu Postdoctoral Science Foundation (No. 0902004C) and Foundation of China University of Mining and Technology (No. 2009A023). 


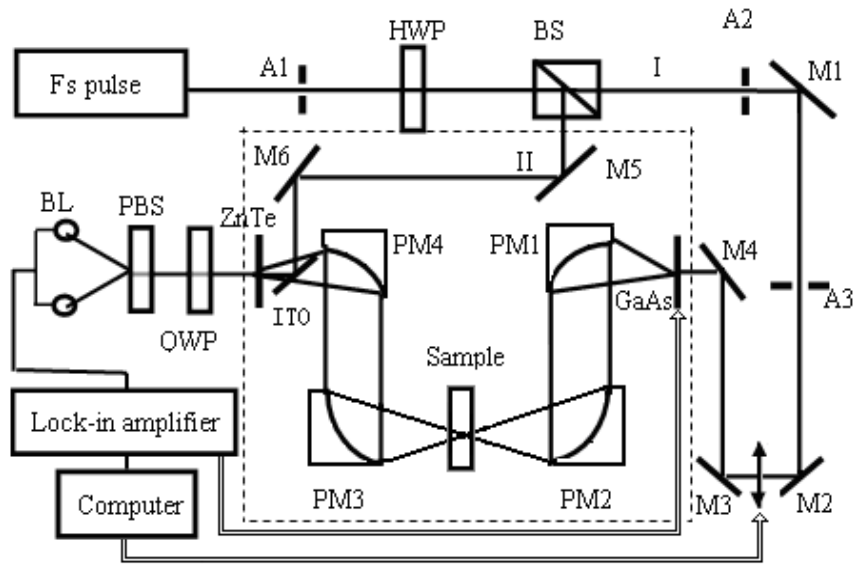

Fig. 1 Schematic diagram of THz-TDS system. BS: beam splitter, PM: parabolic mirror, HWP: half-wave plate, QWP: quarter-wave plate, BL: Balanced photodiode, A: aperture; M: mirror

\section{B. Experiments}

The samples are put between two polyethylene slices. The distance between the two polyethylene slices can be adjusted. In the experiments of this work the distance is $1 \mathrm{~mm}$. Terahertz wave can transmits the polyethylene with little attenuation. The relative humidity in the box in fig, 1 is less than $3.0 \%$ and the environmental temperature is kept at $293 \mathrm{~K}$.

\section{Data analysis method}

The terahertz wave transmitted the sample is measured in this experiment. Sample dispersion and absorption change the field of the transmitted $\mathrm{THz}$ pulse. The relative absorption index is,

$$
A=-\log \left[\left(\frac{A_{\text {sample }}(f)}{A_{\text {reference }}(f)}\right)^{2}\right]
$$

Where $A_{\text {sample }}$ and $A_{\text {reference }}$ is the FFT amplitude of the reference and the sample, they are the function of $f$.

\section{RESULTS AND DISCUSSION}

In figure 2, the real line presents the terahertz wave of reference and the dashed line is that of terahertz wave transmitted through Terylene. From the figure it can be seen that the terahertz wave is attenuated in amplitude and delayed in time. The amplitude is reduced because the Terylene absorbs the terahertz wave, while the wave is delayed because the refractive index of Terylene is bigger than air's.

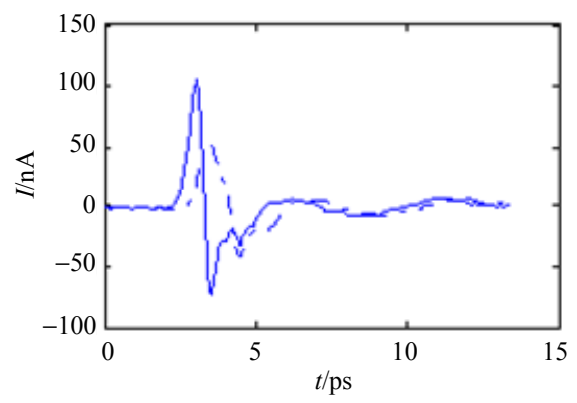

Figure 1. Terahertz wave for reference and Terylene

The terahertz absorption spectra of the synthetic fiber in the experiment can be deduced through Equation (1) and is indicated in Figure 3.
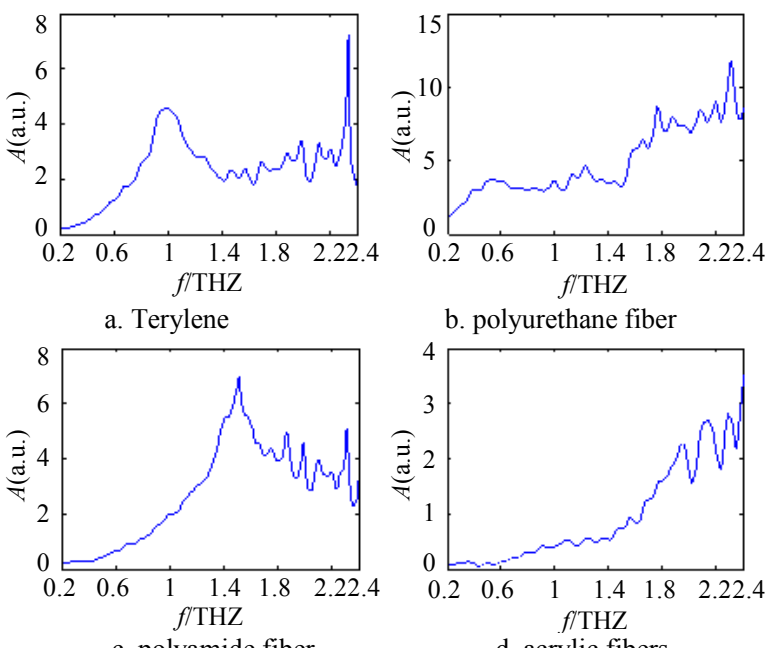

b. polyurethane fiber

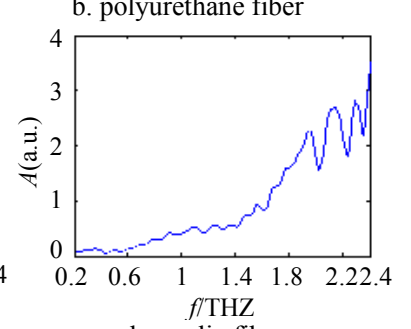

c. polyamide fiber

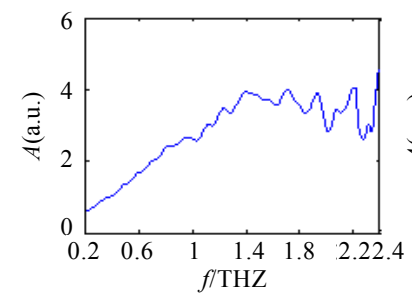

d. acrylic fibers

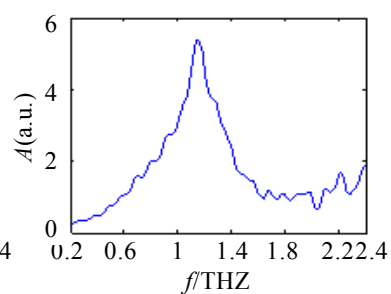

e. tencel

f. vinylon

Figure 2. Terahertz absorption spectra of the synthetic fiber

From the figure, it can be concluded that Terylene, polyamide fiber and vinylon have obvious absorption peaks at $0.98 \mathrm{THz}, 1.51 \mathrm{THz}$ and $1.16 \mathrm{THz}$ respectively. There exists benzene ring and fatty hydrocarbon[1]. Under suitable conditions, macromolecules are apt to crystallize, and the structure of the fibers is tight. There are a lot of amide groups in the molecule chain of polyamide fiber, so polyamide fiber can form strong hydrogen bonds with the amide groups of the adjacent molecule chains. The structure of the molecule of polyamide fiber is regular, has no vast side chains, so it is easy to crystallize when the molecule chain stretch. Vinylon is made of polyvinyl alcohol. After a series of reaction, it can transform 
into polyvinyl formal fiber. Vinylon's degree of orientation and crystallinity are high. Terahertz wave is sensitive to the intermolecular forces. For example, the frequencies of lattice vibration of crystalline and intermolecular hydrogen bonds are situated in terahertz region ${ }^{[15-17]}$. So the absorption peaks of these three fibers are probably the lattice vibration of the crystalline. And the peaks of polyamide fiber may be the vibration of crystalline lattice and the hydrogen bonds. However, polyurethane fiber, acrylic fibers and tencel have no obvious absorption peaks, but their absorption spectra are obviously different. Among the synthetic fibers, polyurethane fiber absorbs terahertz wave the most. Between 06-1.5THz, its absorption index doesn't change much, while above $15 \mathrm{THz}$, it is on the rise. The absorption of acrylic fiber is on the rise, too. The absorption of tencel is different from that of polyurethane fiber, it is growing between $0.2-1.4 \mathrm{THz}$ while above $1.4 \mathrm{THz}$ it change a little. From the analysis above, the absorption of the fibers investigated are different and can be used to distinguish them. Further work is in progress, it includes distinguish synthesis fibers based on their terahertz spectra.

\section{CONCLUSION}

Terahertz absorption spectra of some synthesis fibers are measured with terahertz time domain spectroscopy system. The research results show that their absorption are different obviously. Among them, Terylene, polyamide fiber and vinylon have obvious absorption peaks in terahertz region, and they may be the results of the lattice vibration. The THz spectra of other three fibers all have their own traits. So it is feasible to distinguish these fibers with terahertz time domain spectroscopy. This work provides the basis to differentiate textile fibers based on terahertz technology.

\section{ACKNOWLEDGMENT (HEADING 5)}

We thank the support by Jiangsu Postdoctoral Science Foundation (NO. 0902004C), China Postdoctoral Science
Foundation (NO. 20100481177) and Foundation of China University of Mining and Technology. (NO. 2009A023).

[1] M. YAO. Textile materials science. China Textile \& Apparel Press, Beijing (1980).

[2] W. D. YU. Textile materials science. China Textile \& Apparel Press, Beijing (2006).

[3] W. L. XU. Infrared techniques and textile material. Chemical Industry Press, Beijing (2005).

[4] A Hadni, J. "A short history of 50years of research in the far infared: 1952-2002” Infra. Milli. Waves. Vol. 24, pp. 91-118,2003.

[5] D Abbott. Gallium Arsenide Integrated Circuit (GaAs IC) Symposium, "Directions in TeraHertz technology," Seattle, America, (2002).

[6] K Sakai, Terahertz optoelectronics, Springer, Berlin (2005)

[7] R. A. Cheville, D. Grischkowsky, "Far-infared terahertz time-domain spectroscopy of flames,” Opt. Lett., vol 20, pp. 1646-1648 1995

[8] R. A. Cheville, D. Grischkowsky, "Far-infared foreign and selfbroadened rotational linewidths of high-temprature water vapor," J. Opt. Soc. Amer. A, vol. 16, pp. 317-322,1999.

[9] Y. Q. Chen, H. B. Liu, Y. Q. Deng, "THz spectroscopic investigation of 2,4-dinitrotoluene," Chem. Phys. Lett. Vol. 400, pp. :357-361, 2004.

[10] J. F. Michael, S. Dunja and D. Carroline, "THz spectroscopy of explosives and related compounds," Terahertz for Military and Security Applications II, Proceeding of SPIE, vol. 5411, pp. 84-91, 2004.

[11] C. J. Strachan, P. F. Taday and D. A. Newnham., "Using terahertz pulsed spectroscopy to quantify pharmaceucal polymorphism and crystallinity,” J. Pharm. Sci. vol. 94, pp. 837-846 , 2005.

[12] P. H. Siegel., "Terahertz technology in biology and medicine," IEEE Transactions on Microwave theory and Techniques, vol. 52, pp. 2438$2447,2004$.

[13] B. B. Hu, M. C. Nuss, "Imaging with terahertz waves" Optics Lett., vol. 20, pp. 1716-1718, 1995 .

[14] M. M. Daniel, H. J. Rune, M. C. Nuss, “T-ray imaging,” IEEE Journal of Selected Topics in Quantum Electronics, vol. 2, pp. 679-692,1996.

[15] M. WALTHER, B. M. FISCHER, J. P. UHD, "Noncovalent intermolecular forces in polycrystalline and amorphous saccharides in the far infrared," Chem Phys, vol. 288, pp. 261-268,2003.

[16] A. G. MARKELZ, A. ROITBERF, E. J. HEILWEIL, "Pulsed terahertz spectroscopy of DNA, bovine serum albumin and collagenbetween 0.1 an" Chem Phys Lett, vol. 320, pp. 42-48,2000.

[17] M. WALTHER M, B. FISCHER and M. SCHALL, "Far-infrared vibrational spectra of all-trans, 9-cis and 13-cis retinal measured by $\mathrm{THz}$ time-domain spectroscopy"Chem Phys Lett, 332: 389-395 (2000). 